\title{
The effect of avian blood on Leishmania development in Phlebotomus duboscai
}

\author{
Katerina Pruzinova*, Jan Votypka and Petr Volf
}

\begin{abstract}
Background: The development of pathogens transmitted by haematophagous invertebrate vectors is closely connected with the digestion of bloodmeals and is thus affected by midgut enzymatic activity. Some studies have demonstrated that avian blood inhibits Leishmania major infection in the Old World vector Phlebotomus papatasi; however, this effect has never been observed in the New World vectors of the genus Lutzomyia infected by other Leishmania species. Therefore, our study was focused on the effect of chicken blood on bloodmeal digestion and the development of Leishmania major in its natural vector Phlebotomus duboscai, i.e. in a vector-parasite combination where the effect of blood is assumed. In addition, we tested the effect of avian blood on midgut trypsin activity and the influence of repeated feedings on the susceptibility of sand flies to Leishmania infection.

Methods: Phlebotomus duboscai females were infected by rabbit blood containing L. major and either before or after the infection fed on chickens or mice. The individual guts were checked microscopically for presence and localization of Leishmania, parasite numbers were detected by Q-PCR. In addition, midgut trypsin activity was studied.

Results: Sand fly females fed on chicken blood had significantly lower midgut trypsin activity and delayed egg development compared to those fed on rabbits. On the other hand, there was no effect detected of avian blood on parasite development within the sand fly gut: similar infection rates and parasite loads were observed in $P$. duboscai females infected by L. major and fed on chickens or mouse one or six days later. Similarly, previous blood feeding of sand flies on chickens or mice did not show any differences in subsequent Leishmania infections, and there was equal susceptibility of $P$. duboscai to L. major infection during the first and second bloodmeals.

Conclusion: In spite of the fact that avian blood affects trypsin activity and the oocyte development of sand flies, no effect of chicken blood was observed on the development of L. major in P. duboscai. Our study unambiguously shows that sand fly feeding on avian hosts is not harmful to Leishmania parasites within the sand fly midgut.
\end{abstract}

Keywords: Leishmaniases, Vectors, Bloodmeal digestion, Trypsin, Chicken blood

\section{Background}

Digenetic parasites of the genus Leishmania (Kinetoplastida: Trypanosomatidae) alternate between intracellular amastigotes in mammalian hosts and extracellular promastigotes in sand fly vectors (Diptera: Phlebotominae). In the sand fly vector, development is confined to the digestive tract and is closely connected with bloodmeal digestion (reviewed by $[1,2]$ ).

The source of the bloodmeal influences the digestion and fecundity of females [3-7]. Proteolytic activity in the

\footnotetext{
* Correspondence: katerina.pruzinova@gmail.com

Department of Parasitology, Faculty of Science, Charles University, Prague, Czech Republic
}

(C) 2013 Pruzinova et al.; licensee BioMed Central Ltd. This is an Open Access article distributed under the terms of the Creative Commons Attribution License (http://creativecommons.org/licenses/by/2.0), which permits unrestricted use, distribution, and reproduction in any medium, provided the original work is properly cited.

midgut of haematophagous insects is activated by ingested proteins and the consequent rate of trypsin activity is correlated with the protein content in the bloodmeal $[8,9]$. Thus, the reproductive potential of sand fly females partly depends on the type of bloodmeal and amount of ingested nutrients [4].

Ingested blood affects not only the digestion and fecundity of sand flies but also can affect Leishmania development. Sand fly midgut proteases influence Leishmania development and are one of the obstacles that parasites must overcome to establish an infection in the midgut (reviewed by [1,2] ). Adler [10] first suggested that products of blood serum digestion destroy Leishmania 
parasites in the midguts of 'noncompatible' sand fly species. According to Schlein and Romano [11] and Borovsky and Schlein [12], a specific component of the trypsinlike activity prevents the survival of $L$. donovani in the 'noncompatible' vector Phlebotomus papatasi while the ability to modulate this factor enables L. major to survive in 'compatible' sand fly species. Pimenta et al. [13] described the susceptibility of Leishmania to midgut digestion in the 'compatible' vector $P$. papatasi as stage-specific: L. major amastigotes and fully transformed promastigotes were relatively resistant to $P$. papatasi proteolytic activity, whereas parasites within the amastigote-to-promastigote transition were highly susceptible being killed.

However, even in 'compatible' vectors the bloodmeal from different animals has been described as having different effects on Leishmania [14]. Schlein et al. [15] reported that Leishmania infection is inhibited in its natural vector $P$. papatasi if the sand fly females were fed on turkeys before or after the infection. According to the authors, the parasite reduction is caused by the digestive process and a relatively high DNAase level is induced by nucleated avian erythrocytes. On the other hand, Nieves and Pimenta [16] tested the effect of nine different sources of blood (human, dog, horse, opossum, rodent, chick, chicken, mouse and hamster) on the development of L. braziliensis and $L$. amazonensis in Lutzomyia migonei. The bloodmeal source influenced the infection rates of the females, but none of the bloodmeal types (including avian blood) eliminated Leishmania parasites. Similarly, Sant'Anna et al. [17] noted that chicken blood supports the development of $L$. mexicana in Lutzomyia longipalpis. Moreover, in latestage infections they found similar numbers of metacyclic promastigotes in females infected via rabbit blood or chicken blood [17]. These findings raised the hypotheses that there might be a difference in the effect of avian blood between the New World vectors of the genus Lutzomyia and Old World vectors of the genus Phlebotomus.

Since descriptions of the effects of avian blood on sand fly digestion and Leishmania development are contradictory, we studied the effect of mammalian and avian blood on the trypsin activity and oocyte development of $P$. duboscqi. In parallel experiments we tested whether the digestion of avian blood is harmful to the development of $L$. major in its natural vector: first we repeated the experiments done by Schlein et al. [15] but included proper control groups. Then, to explain our results we compared the susceptibility of $P$. duboscqi to L. major infection acquired in the first or second bloodmeal and in $100 \%$ versus $5 \%$ blood.

\section{Methods}

\section{Sand fly maintenance}

The colony of $P$. duboscqi was maintained under standard conditions as previously described [18].

\section{Fluorometric measurements of trypsin activity}

Trypsin has been reported to affect Leishmania infections in sand flies $[11,12]$. Therefore, we measured trypsin activities after feeding on avian blood. Midguts of P. duboscqi females fed on rabbits or chickens were dissected at 18, 24, 30, 48, and 72 hours post blood meal (PBM) and transferred to $1.5 \mathrm{ml}$ Eppendorf tubes. Each sample contained 10 midguts in $100 \mu \mathrm{l}$ of Tris- $\mathrm{NaCl}(0.1 \mathrm{M}$ Tris, $150 \mathrm{mM}$ $\mathrm{NaCl}, \mathrm{pH}=8.44)$. The samples were homogenised and trypsin activity was measured in 96-well plate by a fluorometric assay with the substrate Boc-Leu-Gly-Arg-AMC (Bachem). Aminomethylcoumarin (AMC) was excited at $355 \mathrm{~nm}$ and fluorescence of released AMC was measured at $460 \mathrm{~nm}$ by a fluorometer (Tecane infinite M200). Data were evaluated statistically using main effect ANOVA (in STATISTICA 6.1 and StatSoft software).

\section{Protein assays of sand fly midgut homogenates}

In bloodsucking insects, levels of proteolytic activity are known to correspond to the quantity and quality of proteins ingested during the bloodmeal $[8,9]$. To explain differences in midgut trypsin activities present after feeding on different blood sources, we measured the protein content of $P$. duboscsqi females. Midguts of P. duboscqi females fed on rabbits or chickens were dissected 4 hours PBM and transferred to $1.5 \mathrm{ml}$ Eppendorf tubes. Each sample contained 15 midguts in $250 \mu \mathrm{l}$ of Tris$\mathrm{NaCl}$ (0.1 M Tris, $150 \mathrm{mM} \mathrm{NaCl}$, pH 7.8). The samples were homogenised and total amounts of midgut protein were quantified according to the Bradford [19] method adapted to 96 -well plates. Ten $\mu$ l of midgut homogenates were mixed with $200 \mu \mathrm{l}$ of the Bio-Rad protein assay reagent diluted $5 x$ in distilled, deionised water. Absorbance was measured in 96-well plate at $595 \mathrm{~nm}$ by the Tecan infinite M200. Bovine serum albumin (Sigma, concentration 1 to $10 \mu \mathrm{g} /$ well) was used as a standard.

\section{Experimental infections of sand flies}

The Leishmania major strain LV561 (LRC-L137; MHOM/ IL/1967/Jericho-II), the same strain as used by Schlein et. al [15], was maintained at $23^{\circ} \mathrm{C}$ on Medium 199 (Sigma) supplemented with $10 \%$ foetal calf serum (Gibco), 1\% BME vitamins (Sigma), 2\% human urine and gentamicin $(80 \mu \mathrm{g} / \mathrm{ml})$.

Females of $P$. duboscqi were fed through a chick-skin membrane on heat-inactivated rabbit blood containing $10^{6}$ promastigotes per $\mathrm{ml}$. If not stated otherwise $100 \%$ rabbit blood was used. Blood-engorged females were separated and maintained on 50\% sucrose. Bloodfed females were always maintained at constant temperature $\left(26^{\circ} \mathrm{C}\right)$ because it is known that ambient temperature affects the digestion and Leishmania development within sand flies [20]. At various intervals post-infection (PI) the individual guts were checked microscopically for the 
presence and localization of Leishmania promastigotes. Parasite loads were graded according to Myskova et al. [21] as light (< 100 parasites/gut), moderate (100-1000 parasites/gut), or heavy (> 1000 parasites/gut). Data were evaluated statistically by means of the $\chi^{2}$ test using $S$ PLUS 2000 software.

The number of Leishmania parasites in individual females was counted using Q-PCR as described previously $[21,22]$. Briefly, experimental females were stored at $-20^{\circ} \mathrm{C}$ and total DNA extraction was performed with a High Pure PCR Template Preparation Kit (Roche) according to the manufacturer's instructions. Q-PCR using Leishmaniaspecific primers (forward: 5'-CTTTTCTGGTCCTCCG GGTAGG-3'; reverse: 5'-CCACCCGGCCCTATTTTAC ACCAA-3' [22]) was performed by the SYBR Green detection method (iQSYBER Green Supermix, Bio-Rad, Hercules, CA) in Bio-Rad iCycler \& iQ Real-Time PCR systems. Statistical evaluation was performed by the Kruskal-Wallis test and Mann-Whitney U-test using STATISTICA 6.1.

\section{The effects of sand fly feeding on avian blood before and after infection}

To evaluate the effect of avian blood on Leishmania infection we followed three different experimental feeding schemes (the first two done according to Schlein et al. [15]): (i) We evaluated the effect of chicken blood taken before infection. Sand fly females fed either on chickens or mice were given a chance to lay eggs in breeding pots and then, after oviposition, were fed an infective bloodmeal (nine days after the first bloodmeal). (ii) In the second scheme we evaluated the effect of avian blood on parasites already present in the gut: females infected with promastigotes in diluted (5\%) blood were fed either on chickens or mice one day PI. The decreased amount of nutrients in the diluted blood resulted in the females having to feed again without laying eggs. (iii) In addition, we evaluated the effect of avian blood during the later phase of Leishmania infection: females infected with promastigotes in diluted (5\% or $10 \%$ ) blood were fed either on chickens or mice six days PI. Initial experiments showed that $10 \%$ blood resulted in higher infection rates, and therefore in repeated experiments we used only this blood concentration.

\section{A comparison of sand fly susceptibility to L. major during the first and second bloodmeal}

One group of $P$. duboscqi females was fed first on noninfected mice, allowed to lay eggs in a breeding pot and then (9 days post first blood feeding) infected experimentally, while the control group (one week younger) was maintained without a bloodmeal until the experimental infection. Both groups were infected simultaneously with the same parasite culture.
The effect of $5 \%$ or $100 \%$ blood in the infective bloodmeal on parasite establishment in the sand fly midgut

To explain the results of infections done using diluted (5\%) blood (experiment (ii)), we compared the infection rates and intensities of infection after feeding on $5 \%$ or $100 \%$ blood. Females were infected with promastigotes in diluted or undiluted rabbit blood and checked on days 1 and 2 PI; by this time infected females had fed on chickens or mice in the previous experiment.

\section{Differences in the digestion of $10 \%$ and $100 \%$ blood}

To explain the results of infections done using diluted (10\%) blood (experiment (iii)), we compared the trypsin activity after feeding on $10 \%$ and $100 \%$ blood. Midguts of females fed on $10 \%$ or $100 \%$ rabbit blood through a chick-skin membrane were dissected at $24,30,48$, and 72 hours PBM and transferred to $1.5 \mathrm{ml}$ Eppendorf tubes. Each sample contained a mixture of 10 midguts in $100 \mu \mathrm{l}$ of Tris- $\mathrm{NaCl}(0.1 \mathrm{M}$ Tris, $150 \mathrm{mM} \mathrm{NaCl}, \mathrm{pH}=8.44)$. Trypsin activity was measured by the fluorometric method described above.

In both groups of females, the time of defecation was also measured. Thirty fully blood-fed females from both groups were individually placed in small glass vials, maintained at $26^{\circ} \mathrm{C}$ and checked twice daily under a binocular microscope for defecation.

\section{Ethical statement}

Animals were maintained and handled in the animal facility of Charles University in Prague in accordance with institutional guidelines and Czech legislation (Act No. 246/1992 coll. on Protection of Animals against Cruelty in present statutes at large), which complies with all relevant European Union and international guidelines for experimental animals. All the experiments (including sand fly feeding) were approved by the Committee on the Ethics of Laboratory Experiments of the Charles University in Prague and were performed under the Certificate of Competency (Registration Number: CZU 246/ 1992, CZ 00177).

\section{Results}

The effect of blood source on midgut trypsin activity Phlebotomus duboscqi females were fed either on rabbits or chickens and dissected at $4,18,24,30,48$, and 72 hours PBM, and protein absorbance and trypsin activity was measured. Data from two independent experiments were pooled. Sand flies fed on chickens had half the midgut protein content compared to those fed on rabbits $(103 \mu \mathrm{g} /$ gut versus $202 \mu \mathrm{g} / \mathrm{gut}$, respectively). Similarly, midgut trypsin activity in females fed on chickens was significantly lower $\left(\mathrm{F}_{(4,50)}=5.26, \mathrm{P}<0.01\right)$. The highest differences between groups were observed during 
the first 24 hours post bloodmeal, with females fed on chickens having 40 - 55\% less trypsin activity in their midguts compared to those fed on rabbits (Figure 1).

\section{The effect of chicken and mice blood on Leishmania development in sand fly midguts}

(i) 9 days before infection: Phlebotomus duboscqi females, previously fed either on chickens or mice, were given an infective bloodmeal nine days later and then dissected on days 2 and 6 PI. Data from three independent experiments were pooled. In both groups of females (fed on chicken versus mouse) similar Leishmania development was observed (Figure 2): no significant differences were found in infection rates or intensities of infection on days 2 and 6 PI (day $2: X^{2}=0.89, P=0.64$; day $\left.6: \chi^{2}=0.10, P=0.76\right)$. On day $6 \mathrm{PI}$, all females from both groups were infected and a majority of them had high parasite loads (over $80 \%$ had heavy infections); Leishmania promastigotes colonized the stomodeal valve in almost all females (99\%). Various promastigote forms, mainly leptomonads and metacyclic promastigotes were present in thoracic midgut.

(ii) 1 day after infection: Females infected with Leishmania promastigotes in 5\% rabbit blood were fed one day later on chickens or mice. Data from three independent experiments were pooled. On days 2, 6, and 9 after the second bloodmeal, no significant differences were observed in Leishmania development: females fed on chickens or mice did not differ in infection rates $\left(\chi^{2}=1.37\right.$, $\mathrm{P}=0.24$ ) or intensities of infection on any of the compared days PI (day 2: $\chi^{2}=2.81, \mathrm{P}=0.42$; day 6: $\chi^{2}=0.19, \mathrm{P}=$ 0.76 ; day $\left.9 X^{2}=3.07, P=0.38\right)$. On day 9 after the second bloodmeal, $50 \%$ of females from both groups were infected; the intensities of infection were high in most of them and

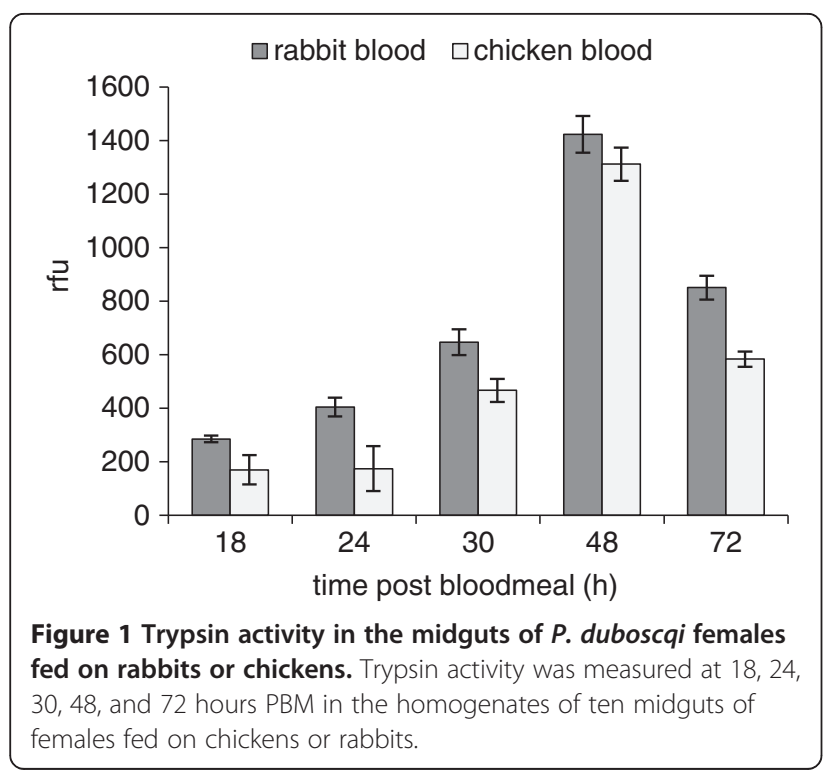

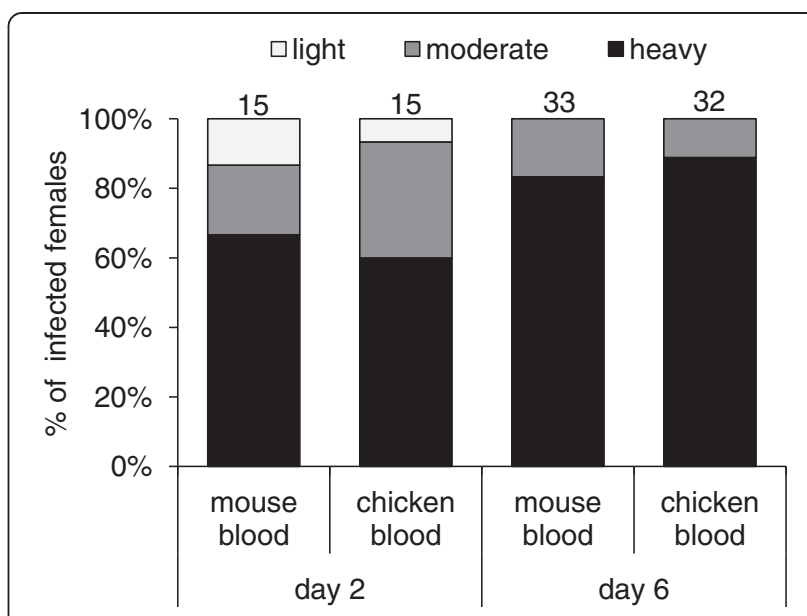

Figure 2 The effect of chicken blood on Leishmania development in sand fly midguts: 9 days before infection. $P$. duboscai females fed either on chickens or mice and nine days later were infected by $10^{6}$ promastigotes per $\mathrm{ml}$ of bloodmeal. Infection rates and intensities of infection were evaluated microscopically in sand fly midguts on days 2 and $6 \mathrm{Pl}$, and infections were classified into three categories: light ( $<100$ parasites/gut), moderate (100-1000 parasites/gut), or heavy (> 1000 parasites/gut). Numbers above the bars indicate the number of dissected females.

promastigotes colonized the stomodeal valve in $100 \%$ of infected females (Figure 3A). Various promastigote forms including metacyclics were observed. Similarly, Q-PCR revealed no significant differences $\left(\mathrm{KW}-\mathrm{H}_{(1 ; 100)}=1.03, \mathrm{P}=\right.$ 0.31 ) in total parasite numbers in sand fly midguts on day 9 after the second bloodmeal (Figure 3B).

(iii) 6 days after infection: Females infected by Leishmania were fed on chickens or mice six days after an infective meal containing $10 \%$ rabbit blood with promastigotes. Data from two independent experiments were pooled. Females from both groups were dissected on days 2 and 6 after the second bloodmeal, and no significant differences were observed between experimental groups (chicken vs. mouse) in infection rates $\left(\chi^{2}=0.05\right.$, $\mathrm{P}=0.82$ ) or intensities of infection (day $2: \mathrm{X}^{2}=5.72, \mathrm{P}=$ 0.13; day 6: $\left.\chi^{2}=0.08, P=0.96\right)$. Leishmania developed similarly in both female groups: infection rates were about $80-85 \%$ and parasite loads were high in a majority of infected females $(50-80 \%)$ (Figure 4A). Promastigotes colonized the stomodeal valve from day 6 after the second bloodmeal in $100 \%$ of infected females. Leptomonad and metacyclic forms prevailed in thoracic midgut. Similarly, Q-PCR revealed no significant differences $\left(\mathrm{KW}-\mathrm{H}_{(1 ; 100)}=\right.$ $1.20, \mathrm{P}=0.27$ ) in total parasite numbers in sand fly midguts on day 6 after the second bloodmeal (Figure 4B).

Sand fly susceptibility to $L$. major infection during the first and second bloodmeals

Leishmania development in females infected by rabbit blood with promastigotes during the first or second 

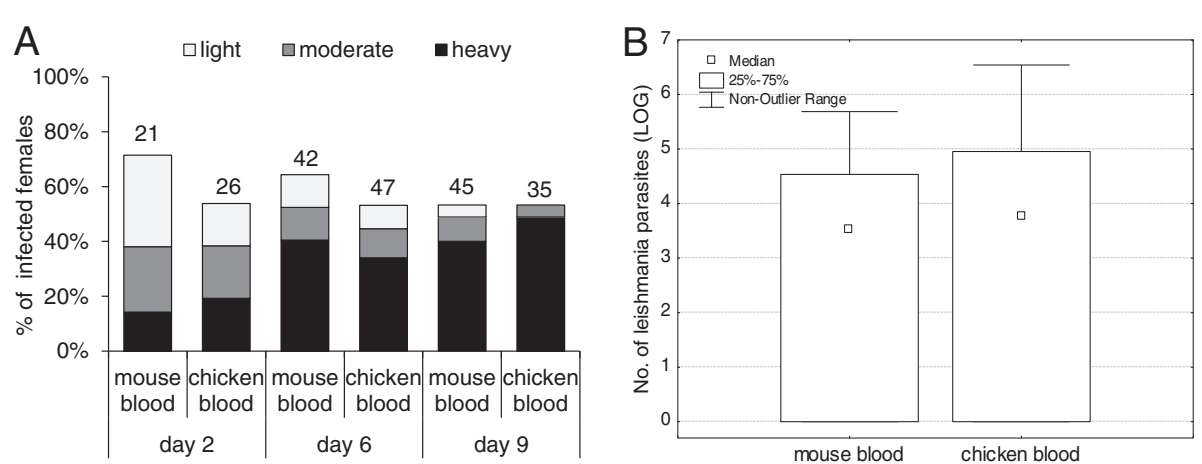

Figure 3 The effect of chicken blood on Leishmania development in sand fly midguts: 1 day after infection. One day after the infection by promastigotes in $5 \%$ rabbit blood $\left(10^{6}\right.$ parasites $\left./ \mathrm{ml}\right)$ P. duboscqi females were allowed to feed on chickens or mice. Infection rates and intensities of infection were evaluated in sand fly midguts on days 2, 6, and 9 after the second bloodmeal. A: Intensities of infection were microscopically classified into three categories: light (< 100 parasites/gut), moderate (100-1000 parasites/gut), or heavy (> 1000 parasites/gut). Numbers above the bars indicate the number of dissected females. B: The precise number of parasites from 50 females of both groups was measured by Q-PCR 6 days after the second bloodmeal.

bloodmeal was compared on days 2, 6, and 9 PI. Data from two independent experiments were pooled. No significant differences were observed between experimental groups $\left(1^{\text {st }}\right.$ vs. $2^{\text {nd }}$ bloodmeal $)$ in infection rates $\left(x^{2}=\right.$ $0.0002, P=0.99$ ) or intensities of infection for any of the compared days PI (day $2: \chi^{2}=3.74, P=0.15$; day $6: x^{2}=$ 3.41, $\mathrm{P}=0.33$ ). In late-stage infections, on days 6 and 9 PI, P. duboscqi females of both groups showed very high infection rates (almost 100\%), high parasite loads (about $80 \%$ of heavy infections with metacyclic forms present in thoracic midgut) and a majority of females had the stomodeal valve colonized by promastigotes (Figure 5 ).

The effect of blood concentration (5\% vs. $100 \%$ ) on parasite establishment in sand fly midguts

In experiments using Leishmania promastigotes in 5\% rabbit blood, infection rates and parasite loads of infected females after the second bloodmeal were lower in comparison with females infected by feeding on $100 \%$ blood. Therefore, we decided to test if the infection rates and parasite loads differ already in the early stage of infection (day 1 and 2), thus before the time of the second feeding (as described in a previous experiment).

Two groups of females were infected by feeding on 5\% or $100 \%$ rabbit blood with promastigotes and were dissected on days 1 and 2 PI. Data from two independent experiments were pooled and significant differences in infection rates $\left(\mathrm{X}^{2}=32.48, \mathrm{P}<0.001\right)$ and intensities of infection in both of compared days PI (day $1: x^{2}=41.92$, $\mathrm{P}<0.001$; day 2: $\left.\mathrm{X}^{2}=51.97, \mathrm{P}<0.001\right)$ were observed between the groups (5\% vs. $100 \%$ blood). While only $65 \%$ of females fed on $5 \%$ blood with promastigotes were infected and the intensities of their infection were usually light or moderate, females fed on $100 \%$ blood were all infected and the intensities of their infections were higher (Figure 6).
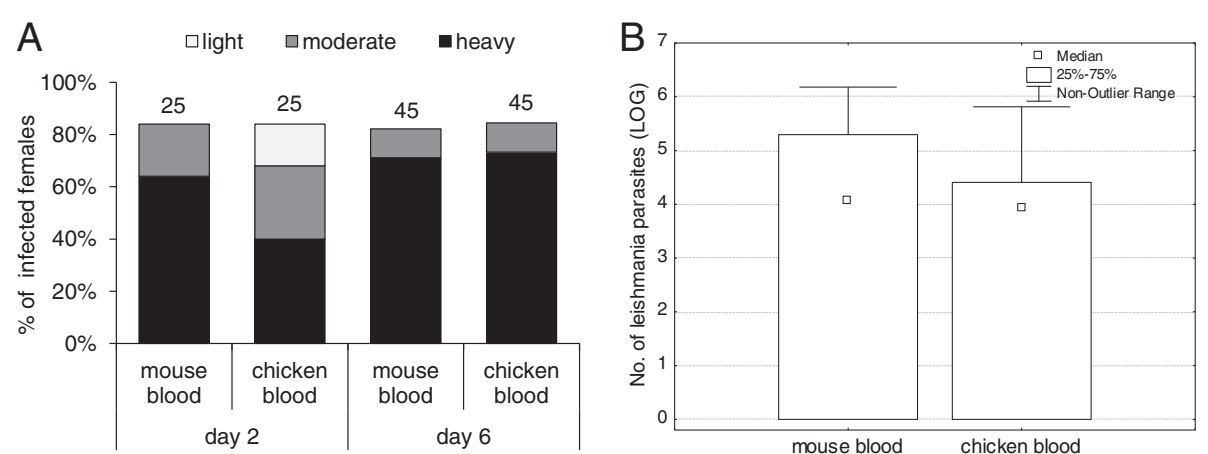

Figure 4 The effect of chicken blood on Leishmania development in sand fly midguts: 6 days after infection. Six days after the infection by promastigotes in $10 \%$ rabbit blood $\left(10^{6}\right.$ parasites $\left./ \mathrm{ml}\right)$ P. duboscai females were allowed to feed on chickens or mice. Infection rates and intensities of infection were evaluated in sand fly midguts on days 2 and 6 after the second bloodmeal. $\mathbf{A}$ : Intensities of infection were microscopically classified into three categories: light (< 100 parasites/gut), moderate (100-1000 parasites/gut), or heavy (>1000 parasites/gut). Numbers above the bars indicate the number of dissected females. B: The precise number of parasites from 50 females of both groups was measured by Q-PCR 6 days after the second bloodmeal. 


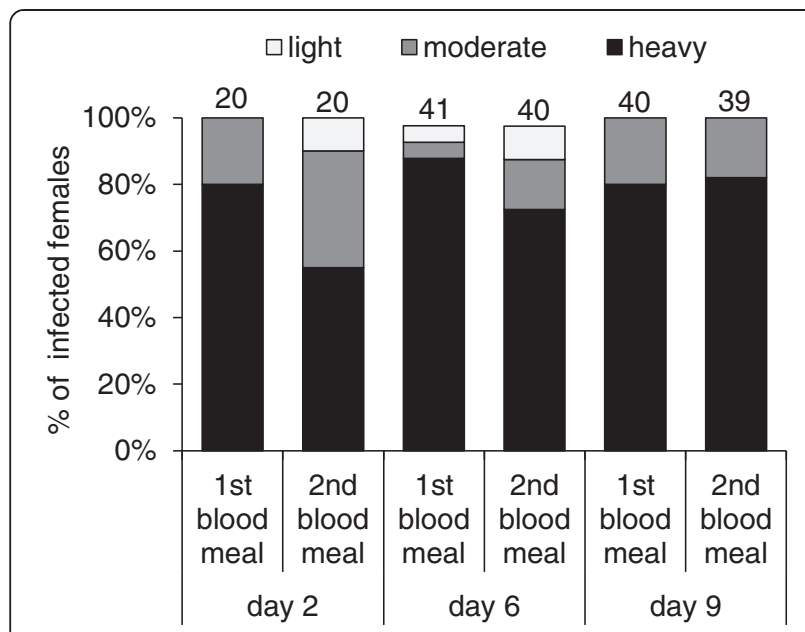

Figure 5 Susceptibility to $L$. major infection during the first and second bloodmeal. P. duboscai females were infected $\left(10^{6}\right.$ promastigotes $/ \mathrm{ml}$ ) during the first or second bloodmeal. Infection rates and intensities of infection were evaluated microscopically in sand fly midguts on days 2,6 , and $9 \mathrm{Pl}$, and infections were classified into three categories: light ( $<100$ parasites/gut), moderate (100-1000 parasites/gut), or heavy (> 1000 parasites/gut). Numbers above the bars indicate the number of dissected females.

\section{Differences in digestion of $10 \%$ and $100 \%$ blood}

In experiments using promastigotes in $10 \%$ rabbit blood, infection rates and parasite loads of infected females after the second bloodmeal were slightly lower in comparison with females infected by feeding on $100 \%$ blood. Therefore, to explain this observation we decided to examine course of trypsin activity and the time of defecation after feeding on diluted or undiluted blood. Data from two independent experiments were pooled. Midgut trypsin activity in females fed on $10 \%$ blood was considerably lower and peaked earlier (Figure 7). The highest values were measured at 30 hours PBM, and by 72 hours PBM was almost zero. In contrast, midgut trypsin activity in females fed on $100 \%$ blood was the highest at 48 hours PMB and at 72 hours PMB was still relatively high. The maximum trypsin activity in females fed on $100 \%$ blood was ten times higher than those fed on $10 \%$ blood (Figure 7).

In addition, we compared the time of defecation: females fed on $10 \%$ blood defecated one or two days earlier compared to those fed on $100 \%$ blood. Females fed on full blood defecated on days $4-5$ post bloodmeal while those fed on $10 \%$ blood defecated on day 3 post bloodmeal. Consequently, this provides considerably less time for Leishmania to escape from the peritrophic matrix and to establish an infection within the sand fly midgut.

\section{Discussion}

Bloodmeals from different animal sources has been reported to affect the digestion, reproductive potential of

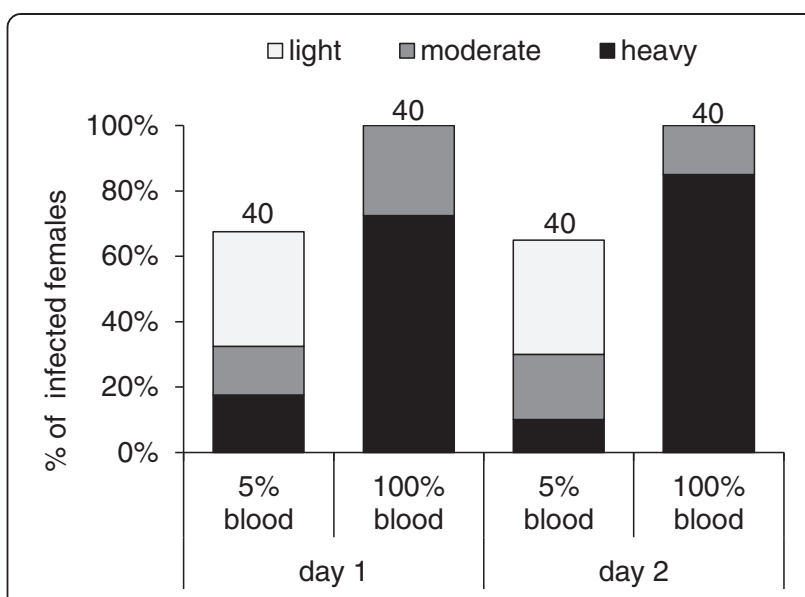

Figure 6 The effect of $5 \%$ or $100 \%$ blood in infective bloodmeals on parasite establishment in the sand fly midgut. P. duboscai females were infected $\left(10^{6}\right.$ promastigotes $\left./ \mathrm{ml}\right)$ by feeding on $5 \%$ or $100 \%$ blood. Sand fly midguts of infected females were investigated microscopically on days 1 and $2 \mathrm{Pl}$, and intensities of infections were classified into three categories: light $(<100$ parasites/gut), moderate (100-1000 parasites/gut), or heavy (> 1000 parasites/gut). Numbers above the bars indicate the number of dissected females.

females and development of Leishmania parasites in the midgut $[3-7,15,23]$. In this study, the effect of chicken blood on digestion, oocyte development and Leishmania infection within the sand fly gut was evaluated.

According to Sant'Anna et al. [17], chicken blood has less than half the total protein of rabbit blood, but the midgut protein content of fully engorged L. longipalpis females fed on rabbit blood was only slightly lower than that of females fed on chicken blood. Clearly, L. longipalpis females were able to partially compensate for the lower protein content in the avian blood source through efficient

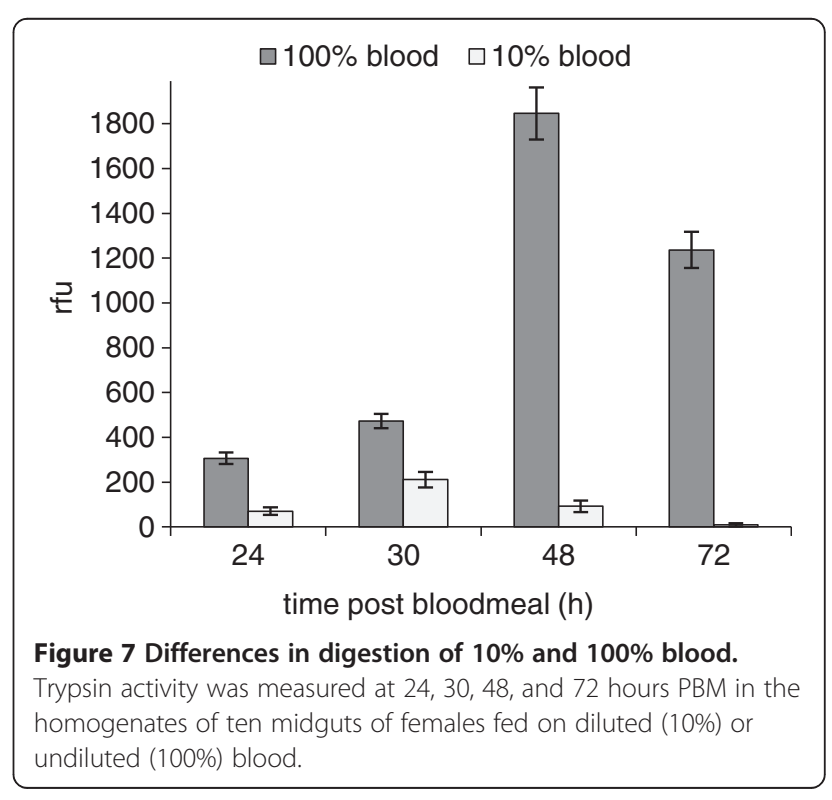


prediuresis [17]. In the experiments presented here $P$. duboscqi females fed on chickens had half the midgut protein content compared to those fed on rabbits, which corresponds to the concentrations measured in chicken and rabbit blood. As prediuresis has also repeatedly been described in P. duboscqi females [24,25], it seems that this species is not able to concentrate avian blood more than rabbit blood.

The lower protein content in the avian blood source influenced the midgut trypsin activity and oocyte development of $P$. duboscqi. Females fed on chickens had significantly lower trypsin activity in the midgut $(18,24,30$, and 72 hours PBM) and slower oocyte development (data not shown) in comparison with females fed on rabbits. These results are consistent with studies on the dependence of enzymatic activity on bloodmeal protein content in mosquitoes, where the proteolytic activity is activated by ingested proteins and that rate of proteolytic activity correlates with protein concentration in the bloodmeal $[8,9]$.

In sand flies, proteins from the bloodmeal are digested for 48-96 hours [26-28], and it is during this time when Leishmania parasites encounter sand fly digestive enzymes. Some authors have shown that the digestion of blood from some hosts may adversely affect the development of Leishmania [14,15,23]. On the other hand, Leishmania was shown to modulate trypsin secretion of the sand fly vector to its own benefit. This effect has been described in the New World (L. longipalpis and $L$. mexicana) [29] as well as in the Old World parasitevector pairs (P. pernicious and L. infantum) [30].

According to Schlein et al. [15] and Schlein and Jacobson [23] digestion of avian blood is harmful to Leishmania parasites within the sand fly midgut. They fed $P$. papatasi females on turkeys or chickens either before or after an infective meal containing rabbit blood with Leishmania promastigotes and in both experimental schemes described a reduction of Leishmania infection [15,23]. In contrast, chicken blood did not reduce the infection of L. braziliensis, L. amazonensis and $L$. mexicana in the New World sand fly species $L$. longipalpis and L. migonei [16,17]. Although Nieves and Pimenta [16] noted a slightly lower percentage of infected females after feeding an amastigote-infected chicken bloodmeal compared to females infected via rodent blood (Cercomys sp.), infections were not eliminated, and L. braziliensis and L. amazonensis established midgut infections. Sant'Anna et al. [17] also did not detect any negative effect of avian blood on L. mexicana infection in the midgut of L. longipalpis; on the contrary, in females infected via chicken blood they reported a trend towards higher infection rates and higher parasite loads in comparison with controls fed on infective rabbit blood [17]. While Sant'Anna et al. [17] used amastigote- initiated infections, in our study the infections were promastigote-initiated.

In the present work the effect of avian blood on the development of $L$. major in $P$. duboscqi was studied using light microscopy and Q-PCR in several experiment schemes where sand fly females were fed on chickens or mice either before or after infection. No significant differences were observed in any of these experiments and we can conclude that digestion of avian blood is not harmful to L. major development either before or after infection. The differences between our and Schlein's results cannot be explained by different techniques or parasite vector pairs. We used the same Leishmania strain (LRC-L137), and P. duboscqi is the sister species of $P$. papatasi within the subgenus Phlebotomus, both being natural vectors of $L$. major $[31,32]$.

Phlebotomus duboscqi females infected using the method of Schlein et al. [15] (infection by promastigotes in $5 \%$ rabbit blood and one day later fed on avian blood) had a relatively low (about 60\%) infection rate in both groups, regardless of whether fed on chickens or mice. However, Schlein et al. [15] tested only the group fed on turkeys before or after infection and did not include any control group fed on a mammalian host. Therefore, their conclusions may have been influenced by the absence of appropriate controls. To confirm this assumption, we studied the effect of diluted blood on Leishmania development in the early stage of infection within the sand fly midgut. While females infected via $100 \%$ blood were all infected with high intensities of infection, females fed on $5 \%$ blood with promastigotes were infected in only $65 \%$ and parasite loads were light or moderate. This experiment revealed that diluted blood in infective meal leads to significantly lower infection rates and parasite loads, probably as a consequence of faster digestion. The peritrophic matrix of $P$. duboscqi females fed on full blood matures in about 12 hours PBM, and its disintegration started only at the third day PBM [33] and females defecated on days 4-5 PBM. On the other hand, in females infected via diluted blood Leishmania promastigotes have a very limited time to escape the peritrophic matrix and establish an infection within the midgut.

To complete the study on the influence of avian blood on Leishmania development we considered the effect of number of feedings and age of females on parasite development within the sand flies. Such effects have previously been described in other bloodsucking arthropods: tsetse flies (Glossina spp.) given trypanosomes in their first bloodmeal were found to be more susceptible to infection compared to flies given trypanosomes in a later bloodmeal [34]. More recent studies by Walshe et al. [35] and Kubi et al. [36] showed that it is rather the age (hours after eclosion) of the flies when they take the first infective bloodmeal or nutritional stress that determines 
the susceptibility to infection. It seems that a higher susceptibility to infection is caused by the physiological immaturity and imperfect immune response of teneral (newly emergent and unfed) tsetse flies [36]. Based on this knowledge, we decided to test the susceptibility of $P$. duboscqi to L. major infection during the first or the second bloodmeal; however, no differences between these two experimental groups of females were observed. This finding corresponds with the lack of any information regarding significant differences in infection rate after the first or the second blood feeding in Nematocera. Such a contrast between brachyceran and nematoceran flies (tsetse and sand flies, respectively) could be explained by differences in bloodmeal digestion mode and the type of peritrophic matrix (PM). Sand flies as well as mosquitoes and other nematoceran haematophagous insects have discontinuous bloodmeal digestion and form PM type 1, while tsetse flies digest blood continuously and form PM type 2 (reviewed by [37]).

\section{Conclusions}

Phlebotomus duboscqi females fed on chicken had lower trypsin activity and slower oocyte development in comparison to those fed on mouse. Importantly, various experiments showed that the feeding of Phlebotomus sand flies on avian blood is not harmful to Leishmania development within their midgut. These experiments indicated that the reduction in Leishmania infection reported by Schlein et al. [15] and Schlein and Jacobson [23] was probably not caused by the inclusion of avian blood but by the experimental scheme using diluted blood.

In addition, the susceptibility of $P$. duboscqi females to L. major infection is equal during the first or the second bloodmeal; the number of feedings or female age did not affect the development of Leishmania.

\section{Competing interests}

The authors declare that they have no competing interests.

\section{Authors' contributions \\ KP performed the experimental work, analysed the data and wrote the manuscript. JV performed and analysed Q-PCR data, performed statistical analysis. PV designed the study, contributed to interpretation and wrote the manuscript. All authors read and approved the final version of the manuscript}

\section{Acknowledgements}

We thank Lucie Ječná for help during experimental infections. The study was partially supported by EU grant 2011-261504 EDENext and the paper is cataloged by the EDENext Steering Committee as EDENext 165.

Received: 22 July 2013 Accepted: 27 August 2013

Published: 2 September 2013

\section{References}

1. Kamhawi S: Phlebotomine sand flies and Leishmania parasites: friends or foes? Trends Parasitol 2006, 22:439-445.

2. Dostalova A, Volf P: Leishmania development in sand flies: parasite-vector interactions overview. Parasit Vectors 2012, 5:276.
3. Ward RD: The colonization of Lutzomyia flaviscutellata (Diptera: Psychodidae), a vector of Leishmania mexicana amazonensis in Brazil. J Med Entomol 1977, 14:469-476.

4. Ready PD: Factors affecting egg production of laboratory-bred Lutzomyia longipalpis (Diptera: Psychodidae). J Med Entomol 1979, 16:413-423.

5. Benito-De Martin MI, Gracia-Salinas MJ, Molina-Moreno R, Ferrer-Dufol M, Lucientes-Curdi J: Influence of the nature of the ingested blood on the gonotrophic parameters of Phlebotomus perniciosus under laboratory conditions. Parasite 1994, 1:409-411.

6. Hanafi HA, Kanour WW, Beavers GM, Tetreault GE: Colonization and bionomics of the sandfly Phlebotomus kazeruni from Sinai, Egypt. Med Vet Entomol 1999, 13:295-298.

7. Noguera P, Rondon M, Nieves E: Effect of blood source on the survival and fecundity of the sandfly Lutzomyia ovallesi Ortiz (Diptera: Psychodidae), vector of Leishmania. Biomedica 2006, 26(Suppl 1):57-63.

8. Briegel H, Lea AO: Relationship between protein and proteolytic activity in the midgut of mosquitoes. J Insect Physiol 1975, 21:1597-1604.

9. Felix CR, Betschart B, Billingsley PF, Freyvogel TA: Post-feeding induction of trypsin in the midgut of Aedes aegypti L (Diptera, Culicidae) is separable into 2 cellular-phase. Insect Biochem 1991, 21:197-203.

10. Adler S: Factors determining the behaviour of Leishmania sp. in sandflies. Harefuah 1938, 14:1-6.

11. Schlein $Y$, Romano H: Leishmania major and L. donovani: effects on proteolytic enzymes of Phlebotomus papatasi (Diptera, Psychodidae). Exp Parasitol 1986, 62:376-380.

12. Borovsky D, Schlein Y: Trypsin and chymotrypsin-like enzymes of the sandfly Phlebotomus papatasi infected with Leishmania and their possible role in vector competence. Med Vet Entomol 1987, 1:235-242.

13. Pimenta PF, Modi GB, Pereira ST, Shahabuddin M, Sacks DL: A novel role for the peritrophic matrix in protecting Leishmania from the hydrolytic activities of the sand fly midgut. Parasitology 1997, 115:359-369.

14. Adler S: Leishmania. Adv Parasitol 1964, 2:35-96.

15. Schlein Y, Warburg A, Schnur LF, Shlomai J: Vector compatibility of Phlebotomus papatasi dependent on differentially induced digestion. Acta Trop 1983, 40:65-70.

16. Nieves E, Pimenta PF: Influence of vertebrate blood meals on the development of Leishmania (Viannia) braziliensis and Leishmania (Leishmania) amazonensis in the sand fly Lutzomyia migonei (Diptera: Psychodidae). Am J Trop Med Hyg 2002, 67:640-647.

17. Sant'Anna MRV, Nascimento A, Alexander B, Dilger E, Cavalcante RR, DiazAlbiter HM, Bates PA, Dillon RJ: Chicken blood provides a suitable meal for the sand fly Lutzomyia longipalpis and does not inhibit Leishmania development in the gut. Parasit Vectors 2010, 3:3.

18. Volf $P$, Volfova $V$ : Establishment and maintenance of sand fly colonies. J Vector Ecol 2011, 36(Suppl 1):S1-S9.

19. Bradford MM: A rapid and sensitive method for the quantitation of microgram quantities of protein utilizing the principle of protein-dye binding. Anal Biochem 1976, 72:248-254.

20. Hlavacova J, Votypka J, Volf P: The effect of temperature on Leishmania development in sand flies. J Med Entomol 2013, 50:935-938.

21. Myskova J, Votypka J, Volf P: Leishmania in sand flies: comparison of quantitative polymerase chain reaction with other techniques to determine the intensity of infection. J Med Entomol 2008, 45:133-138.

22. Mary C, Faraut F, Lascombe L, Dumon H: Quantification of Leishmania infantum DNA by real-time PCR assay with high sensitivity. J Clin Microbiol 2004, 42:5249-5255.

23. Schlein $Y$, Jacobson RL: Some sandfly food is a Leishmania poison. Bull Soc Vector Ecol 1994, 19:82-86.

24. Sadlova J, Reishig J, Volf P: Prediuresis in female Phlebotomus sandflies (Diptera : Psychodidae). Eur J Entomol 1998, 95:643-647.

25. Sadlova J, Volf P: Occurrence of Leishmania major in sandfly urine. Parasitology 1999, 118:455-460.

26. Dillon RJ, Lane RP: Bloodmeal digestion in the midgut of Phlebotomus papatasi and Phlebotomus langeroni. Med Vet Entomol 1993, 7:225-232.

27. Volf $P$, Killick-Kendrick R: Post-engorgement dynamics of haemagglutination activity in the midgut of phlebotomine sandflies. Med Vet Entomol 1996, 10:247-250.

28. Telleria EL, de Araujo AP, Secundino NF, d'Avila-Levy CM, Traub-Cseko YM: Trypsin-like serine proteases in Lutzomyia longipalpis - expression, activity and possible modulation by Leishmania infantum chagasi. PLoS One 2010, 5:e10697. 
29. Sant'Anna MRV, Diaz-Albiter H, Mubaraki M, Dillon RJ, Bates PA: Inhibition of trypsin expression in Lutzomyia longipalpis using RNAi enhances the survival of Leishmania. Parasit Vectors 2009, 2:62.

30. Dostalova A, Votypka J, Favreau AJ, Barbian KD, Volf P, Valenzuela JG Jochim RC: The midgut transcriptome of Phlebotomus (Larroussius) perniciosus, a vector of Leishmania infantum: comparison of sugar fed and blood fed sand flies. BMC Genomics 2011, 12:223.

31. Lawyer PG, Ngumbi PM, Anjili CO, Odongo SO, Mebrahtu YB, Githure J, Koech D, Roberts CR: Development of Leishmania major in Phlebotomus duboscqi and Sergentomyia schwetzi (Diptera: Psychodidae). Am J Trop Med Hyg 1990, 43:31-43.

32. Cihakova J, Volf P: Development of different Leishmania major strains in the vector sandflies Phlebotomus papatasi and P. duboscqi. Ann Trop Med Parasitol 1997, 91:267-279.

33. Sadlova J, Volf P: Peritrophic matrix of Phlebotomus duboscqi and its kinetics during Leishmania major development. Cell Tissue Res 2009, 337:313-325.

34. Welburn SC, Maudlin I: The nature of the teneral state in Glossina and its role in the acquisition of trypanosome infection in tsetse. Ann Trop Med Parasitol 1992, 86:529-536.

35. Walshe DP, Lehane MJ, Haines LR: Post eclosion age predicts the prevalence of midgut trypanosome infections in Glossina. PLoS One 2011, 6:e26984.

36. Kubi C, Van den Abbeele J, De Deken R, Marcotty T, Dorny P, Van den Bossche P: The effect of starvation on the susceptibility of teneral and non-teneral tsetse flies to trypanosome infection. Med Vet Entomol 2006, 20:388-392.

37. Lehane MJ: Peritrophic matrix structure and function. Annu Rev Entomol 1997, 42:525-550.

doi:10.1186/1756-3305-6-254

Cite this article as: Pruzinova et al: The effect of avian blood on

Leishmania development in Phlebotomus duboscqi. Parasites \& Vectors $20136: 254$

\section{Submit your next manuscript to BioMed Central and take full advantage of:}

- Convenient online submission

- Thorough peer review

- No space constraints or color figure charges

- Immediate publication on acceptance

- Inclusion in PubMed, CAS, Scopus and Google Scholar

- Research which is freely available for redistribution 